\title{
Islam militant et nouvelles formes de mobilisation culturelle
}

Amel Boubekeur

\section{(2) OpenEdition}

1 Journals

Édition électronique

URL : http://journals.openedition.org/assr/9333

DOI : $10.4000 /$ assr.9333

ISSN : 1777-5825

Éditeur

Éditions de l'EHESS

\section{Édition imprimée}

Date de publication : 1 septembre 2007

Pagination : 119-138

ISBN : 978-2-7132-2144-6

ISSN : 0335-5985

Référence électronique

Amel Boubekeur, «Islam militant et nouvelles formes de mobilisation culturelle », Archives de sciences sociales des religions [En ligne], 139 | juillet - sepembre 2007, mis en ligne le 19 novembre 2010 ,

consulté le 10 décembre 2020. URL : http://journals.openedition.org/assr/9333 ; DOI : https://doi.org/ 10.4000/assr.9333 


\section{Amel Boubekeur}

\section{Islam militant et nouvelles formes de mobilisation culturelle}

Pendant l'année 2004-2005, la diffusion, en France, de la chaîne du Hizbollah libanais, al Manar, a représenté pour l'ensemble des médias le symbole de la radicalisation islamique supposée des musulmans de France à travers l'importation d'un modèle islamiste moyen-oriental. Pourtant, au même moment, c'est une toute autre mise en scène de l'identité islamique que les musulmans engagés ${ }^{1}$ pratiquaient sur l'ensemble du continent européen et aux États-Unis. Sur l'hexagone, par exemple, loin des images sanglantes du jihad et de l'obsession du combat "contre les ennemis de l'islam occidentaux", les jeunes musulmans recherchaient plutôt l'ambiance d'un islam "cool », libéré des stigmates des anciennes rhétoriques islamistes ${ }^{2}$. Lors des soirées du congrès annuel de l'Union des organisations islamiques de France au Bourget, en mars 2005, ou bien encore lors de la tournée nationale des concerts islamiques ${ }^{3}$ "Rhythm' N' Nasheed " en décembre 2004, on pouvait entendre chanter des imams au look de crooners pieux, guitare à la main, faisant se soulever des centaines de jeunes garçons et

1. Les musulmans engagés, c'est principalement d'eux dont il s'agira dans cet article, recourent à un argumentaire social de définition de soi marqué par une religiosité islamique publique. Souvent, leur socialisation se fait au contact d'acteurs de la ré-islamisation en Occident, majoritairement venus des pays musulmans et parfois proches de la pensée islamiste, tels que les imams, les leaders associatifs ou les conférenciers (la réislamisation désigne le fait de vivre une socialisation religieuse non plus héritée des parents musulmans mais acquise de façon volontariste à travers la fréquentation de mouvements religieux, de lieux de culte, de groupes de pairs telles que les associations de jeunes musulmans...). On considérera ces nouveaux acteurs comme les héritiers historiques de l'islamisme, bien qu'ils cherchent à s'en différencier. Pour une approche du lien entre le nouvel esprit de l'islam engagé et l'idéologie historique de l'islamisme voir Boubekeur (2006).

2. L'islamisme est un mouvement historiquement né dans le monde musulman. Il recouvre aujourd'hui un ensemble de tendances qui ont en commun « l'utilisation politique et militante de la religion ». Cette définition s'inspire de celle de H. Laurens (2000). Elle est citée par M. BennaniChraïbi et O. Fillieule (2003). Les principales revendications de ce mouvement furent l'imposition de mœurs nouvelles à la société civile et le renversement de l'équilibre des forces politiques dans ces pays, notamment à travers la demande d'édification d'un État islamique. L'islamisme, tel que nous l'entendons ici, n'englobe pas les actions violentes, type attentats kamikazes, revendiquées depuis le 11-septembre.

3. Islamique désigne le fait de vouloir explicitement situer ses actions par rapport à l'islam. 
filles au son des louanges à Dieu, à la manière des gospels américains, les bras se balançant dans le ciel, briquets allumés, demandant des autographes et hurlant des takbîr ("Allahu Akbar", Dieu est le plus grand) d'encouragement aux artistes sur scène...

Le militantisme politique islamiste a été pendant longtemps le moyen principal proposé aux musulmans engagés pour se mobiliser autour de l'islam. Aujourd'hui, nombreux sont ceux qui délaissent les meetings et regroupements politiques de l'islamisme classique. Ils choisissent la société du spectacle islamique ${ }^{4}$ comme nouveau répertoire d'action (Tilly, 1978) ${ }^{5}$ permettant de militer " islamiquement ", de parler de leur histoire, de leurs causes et de leurs droits. Que nous apprend cette tension entre l'islamisme classique et ces nouvelles formes de mobilisation culturelles et artistiques, à propos des transformations récentes du militantisme des musulmans engagés, ainsi que sur le devenir de la charge politique de l'islamisme ? Le recours à cette société du spectacle islamique par les musulmans engagés représente-t-il simplement l'émergence d'une sous-culture populaire américanisée liée à la "mondialisation » de l'islam (Roy, 2002), ou traduit-il plus profondément un renouvellement du militantisme avec ses conséquences politiques et identitaires ?

Son analyse nous permet, en tout cas, de cerner au moins trois mutations fondamentales de l'identité post-islamiste d'aujourd'hui en Occident (Roy, Haënni, 1999) ${ }^{6}$. La première tient à la manière dont les musulmans engagés recourent à ces nouvelles formes d'expression et d'intervention publiques de l'identité islamique, avec la volonté de casser les clichés qui lui sont accolés. La seconde montre un retour et une participation au sein de l'espace public global, avec la volonté de se débarrasser de certaines contraintes normatives et idéologiques de confinement. Une des nouveautés majeures amenées par les acteurs de la société du spectacle islamique telle qu'elle nous intéresse dans cet article, est en

4. Le titre est emprunté à l'ouvrage de Guy Debord (1967). Il y étudie la façon dont une idéologie (en l'occurrence le capitalisme) se met en scène comme source du bien. Certains aspects de cette problématique nous semblent tout à fait convenir aux enjeux actuels de l'islam engagé. Plus spécifiquement, on désignera par société du spectacle islamique l'ensemble des supports scéniques non traditionnels de prédication, d'affiliation, de diffusion de normes et de valeurs, utilisés par les élites islamiques tels que la pop et le rap, les sketchs et le théâtre, la télévision, les bandes dessinées, les radios et chaînes de télévision ainsi que certaines formes de transmission du savoir islamique comme les conférences.

5. Charles Tilly nomme "répertoire d'action collective ", la palette d'actions dont usent les groupes protestataires. Ce répertoire d'action peut évoluer à travers le temps, en fonction du message et de l'identité que le groupe souhaite défendre ou encore face à l'environnement dans lequel il se trouve.

6. Le post-islamisme désigne le cadre historique hérité de l'islamisme après l'échec de ce dernier, principalement concernant l'islamisation du champ politique et de l'État. Pour Asaf Bayat, créateur du terme, il est caractérisé par une préséance des " rights instead of duties, plurality in place of a singular authoritative voice, historicity rather than fixed scriptures, and the future instead of the past» (Bayat, 2005). 
effet de vouloir être dans l'espace public global tout en maintenant de façon non revendicative et non oppositionnelle une identité musulmane engagée. Enfin, les acteurs de cette société du spectacle islamique innovent dans la gestion de l'héritage des codes de l'islamisme, qui furent souvent à la base de la construction des communautés musulmanes engagées. Ils portent alors un regard réflexif sur l'islamisme qui devient une forme de tradition, nous verrons plus loin en quels termes.

\section{De nouvelles pratiques dans l'espace public}

Encore marginal il y a quelques années, le nombre des concerts, sketches, pièces de théâtre et festivals culturels islamiques d'un nouveau genre augmente sans cesse. Cette émergence d'une véritable SSI (société du spectacle islamique) ne semble cependant pas entrer dans le débat public concernant l'islam. Devant la contradiction de définitions par le haut de ce qu'est et/ou doit être l'islam (identité islamique confisquée par une pluralité d'acteurs qui peuvent aussi bien être responsables associatifs, leaders d'opinions, journalistes, chercheurs en sciences sociales, musulmans ou non...), et les pratiques nouvelles qui émergent, la question est aujourd'hui de savoir dans quelles configurations s'organise toute pratique publique et engagée, avec pour point de départ l'islam.

Penser que cette pratique s'inscrit exclusivement dans un schème islamiste ou au contraire, qu'elle s'en détache complètement, serait nier l'existence de dynamiques historiques de réappropriation de l'identité islamique et les logiques différentielles de mobilisation qu'elles engendrent chez les musulmans engagés. Pour comprendre cette tension entre cet héritage historique et la nécessité de réinventer des pratiques de mobilisation, il faut considérer ce qu'est le capital islamique $^{7}$ des musulmans engagés nés en Occident. Il est composé de références aussi bien liées à la culture occidentale laïque, à la culture des parents qu'à la socialisation islamique qu'ils ont connue lors de leur réislamisation. Les pratiques proposées par la SSI puisent dans cette base commune et traduisent chez les musulmans engagés un effort de prise en charge de leur identité. Celle-ci n'est plus subie mais rationalisée. Elle se réorganise selon la place qu'ils entendent occuper dans l'espace public global en apportant une voix différente car islamique, mais qui n'empêche plus de se positionner sur des valeurs non spécifiques à l'islam et sur la question du bien commun. Cette réappropriation n'est pas forcément le fait de réaménagements institutionnels ou centralisés par les associations islamiques, mais semble plutôt prendre la voie d'un usage social de l'islamisme assez différencié de sa promulgation idéologique première. Aujourd'hui, pour les musulmans engagés, convaincre leurs adversaires du bien-fondé de leur choix

7. On entend par capital islamique, la somme commune de codes (manière de s'habiller, de se saluer, relations hommes-femmes...), et d'expériences (historiques : le 11-septembre, les polémiques autour du voile; quotidiennes : fréquenter les mêmes conférences, subir les mêmes disqualifications...) spécifiques à l'histoire des musulmans engagés en Occident. 
religieux et revendiquer leur appartenance privilégiée à la communauté restent des préoccupations importantes. Cependant, on assiste à travers l'art et la culture, à une recomposition originale de ces repères hérités de l'histoire commune islamique, dont l'islamisme fait partie.

Ainsi à travers la SSI, les anciens motifs de prédication des sermons autoritaires et culpabilisants laissent place aux pastiches corrosifs sur les défauts de la Oumma (la communauté des croyants) d'un one man show islamique. Les anciens modes d'affiliation associative prônant les réunions hebdomadaires et le soutien scolaire des enfants se voient concurrencés par des rappels spirituels sur des mélodies que l'on peut écouter en faisant son jogging. L'idée de retrouver ses amis engagés autour de la création d'un DVD, de la réalisation d'une peinture ou encore de l'invention d'un poème s'inspirant de la cause à défendre, devient plus séduisante que celle de se mobiliser à travers des manifestations de rue.

L'émergence d'une SSI permet aussi une redéfinition de l'identité pour capter l'attention publique différemment. C'est en grande partie le changement de cadre de l'actualité, le 11-septembre ou encore les polémiques sur la question du voile en France, qui poussent à cette réadaptation ${ }^{8}$. Il est difficile désormais de s'identifier à un islam engagé si on souhaite le faire à travers l'opposition, la tension ou la polémique. La SSI est un moyen de combattre les discours publics stigmatisant ou archaïsant l'islam. Elle permet de renouveler l'engagement militant de beaucoup de musulmans, déçus autant par les définitions identitaires qui leur étaient proposées dans l'espace public global à travers le profil du " beur laïc " que par l'obsession islamiste pour le profil du militant intellectuel qui avait occupé, jusqu'à la fin des années 1990, les fonctions de mise en échec des stigmates dont la SSI prend désormais la relève.

La culture islamique va devenir une ressource positive pour envisager les questions de transmission et de reproduction de l'identité du groupe des musulmans engagés dont l'histoire est mouvementée et fragmentée (Boubekeur, 2005a ; 2005 b). Les frontières de l'intégration, la consolidation du groupe ne sont plus seulement basées sur le corpus religieux normatif (entre bons et mauvais observants), mais aussi sur la construction d'une culture islamique de prestige, dont il faut maîtriser les codes, en grande partie sécularisés et calqués sur les canons esthétiques de l'Occident, sur son libéralisme, son efficacité et sa compétitivité. Il ne suffit plus pour les chanteurs de la SSI de chanter Dieu «avec une bonne niyya (une intention religieuse sincère) ", mais il faut également que les pochettes de leurs albums aient un design dernier cri, le gage suprême de qualité étant désormais de passer sur Skyrock (radio française pour adolescents) ou sur HBO

8. Cependant ne nous méprenons pas : la créativité islamique n'est pas uniquement réflexive (épousant le cadre d'un certain déterminisme social), mais traduit aussi l' « habilitation » des nouveaux acteurs islamiques à travers l'autonomisation de leurs références. 
(chaîne câblée américaine très à la mode), ou encore d'être produit par une grande maison de disque pour ne plus toucher uniquement un public musulman.

La SSI remet également en cause les qualifications qui donnaient autrefois le droit d'agir publiquement sur le thème de l'islam. La représentativité ne se fait plus sur la logique du nombre de militants. Plus encore, la stratification du mouvement social islamiste en Occident ne se fait plus exclusivement par la classe sociale et le capital économique (Khosrokhavar, 1997 ; Kepel, 2000) ${ }^{9}$ mais aussi à travers le goût, la culture, amenant un registre de mobilisations plus civilisationnelles, l' "Islamic way of life » (Göle, 2002), que politiques. La SSI peut être envisagée comme un espace de socialisation pour les musulmans engagés en Occident, un espace ressource disponible qui ne demande plus l'exhaustivité à ses membres : ces derniers sont libres de consommer, d'avoir des partenariats ou une audience en dehors de la communauté engagée. La SSI répond à l'un des enjeux majeurs post-11-septembre : définir ce que doit être un musulman après l'islamisme et gérer son héritage pluriculturel.

\section{La Société du Spectacle Islamique en France}

Au début des années 1980, une nouvelle génération d'intellectuels islamistes issus du monde arabe (Kepel, Richard, 1990) et appartenant à la réorganisation des mouvements islamistes de masses post-indépendance, prend en charge une bonne part de la réislamisation des jeunes musulmans nés en France. Ces militants associatifs exilés, principalement issus de la mouvance des Frères Musulmans, amènent avec eux leur expérience des activités artistiques militantes calquées sur le schéma islamiste des années 1970. Ils avaient constitué des groupes de nachîd (chants religieux militants) dans leur pays d'origine (ceci est particulièrement vrai pour les Tunisiens du Mouvement de la Tendance Islamique fondé par Rachid Ghanoushi) et les mettent à la disposition des jeunes musulmans nés en France. Ceux-ci commencent à les écouter, voire à composer eux-mêmes des nachîd glorifiant la Oumma et sa solidarité, la résistance du peuple palestinien. Ils vont jusqu'à mettre en scène des pièces de théâtre sur les maux du monde musulman en décadence... Néanmoins, ces activités se tenant souvent dans les mosquées, elles étaient réservées à un public d'initiés et elles restaient le fait de l'élite islamiste arabophone exilée.

Dans les années 1990, la réappropriation de ces méthodes se fait selon l'histoire et les codes particuliers de la génération de jeunes musulmans nés en France. Les thèmes ne sont plus les mêmes, et si les louanges à Dieu restent présentes, elles

9. Dans les années 1990, le recours à l'islam était pensé comme résultant de l'exclusion sociale et la mobilisation islamiste comme basée sur une répartition des rôles en termes de classes sociales telles que dans les pays arabes la bourgeoisie pieuse, la jeunesse urbaine pauvre, et l'intelligentsia militante. 
se font désormais en français et affirment que "même si tu t'appelles Christian tu peux être musulman ${ }^{10}$. Le rap islamique (Amghar, 2003) utilise une musique plus rythmée que les percussions traditionnelles arabes pour ses premiers prêches annonçant entre autres "la fin $d u$ monde " ${ }^{11}$ et l'apocalypse des banlieues. Les pièces de théâtre deviennent des sketches dont certaines associations, comme les Jeunes Musulmans de France ${ }^{12}$, se font les spécialistes. Elles mettent en scène "des brigades anti-chaytan (anti-démon) » traquant l'alcool, les flirts et la fréquentation des boîtes de nuit chez les "frères ». Cependant, durant cette période, les modes de fabrication et de diffusion restent encore assez amateurs. Le groupe star et précurseur du nachîd de l'islam de France, "le Silence des Mosquées ", ne donne pas de concert public, ses cassettes sont vendues, et surtout piratées, sur les marchés de banlieues et les visages des musiciens restent inconnus de la population ré-islamisée en France. Ces supports artistiques, restent donc cantonnés à la vie fermée militante de groupe.

Au début des années 2000, cette militance s'individualise. Beaucoup de militants ré-islamisés proches des élites islamistes exilées paient le prix de leur identification à ce groupe par leur marginalisation (tant dans le champ politique que social ou universitaire). Ils décident donc de prendre certaines distances avec la communauté des "frères et sours", se recentrant sur leurs objectifs personnels d'accomplissement de soi. Ils contestent également de plus en plus les cadres d'action islamique imposés par ceux qu'ils appellent les "arabophones ", c'està-dire la première génération d'islamistes venus du monde musulman. Cela va générer d'autres attentes tant chez le public que chez les artistes de la SSI. En effet, les grands responsables associatifs se partageant la gestion du paysage islamique français échouent à fédérer en une seule et même orientation leurs anciens sympathisants. Les jeunes musulmans nés en France ayant fréquenté ces mouvements, ne souhaitent plus y être cantonnés, et tentent de trouver d'autres moyens pour acquérir la ressource islam.

Le début des années 2000 doit donc gérer l'héritage islamiste de l'utilisation de la culture, et ce sont principalement des jeunes "francophones » issus de la mouvance française des Frères Musulmans, qui sont à l'avant-garde du renouveau

10. Refrain d'un des succès dans les années 1990 du groupe phare «Le Silence des Mosquées ». Composé de deux étudiants dijonnais ayant fréquenté l'institut d'études islamiques de ChâteauChinon, c'est le premier groupe de nachîd francophone écouté sur l'ensemble du territoire, marquant profondément les premières générations de jeunes ré-islamisés.

11. Morceau du groupe strasbourgeois NAP (New African Poets) dont faisait partie le converti Abd al Malik (2004).

12. Fédération nationale d'associations de jeunes affiliée à l'UOIF, l'Union des organisation islamique de France, une des grandes fédérations d'associations islamiques en France. JMF a longtemps été présidée par Farid Abdelkrim, ancien membre du conseil d'administration de l'UOIF et actuel responsable de la production artistique de GEDIS, le département des productions audio/vidéo de l'UOIF. JMF fut parmi les principaux animateurs des regroupements islamiques tels que le Bourget, les journées culturelles ou les conférences, à travers des sketchs et pièces de théâtre. 
culturel islamique. Celui-ci se fait hors des codes de l'islamisme traditionnel des années 1980 et 1990, basés sur le repli sur une audience d'élus, l'austérité, le contrôle des corps et surtout l'authenticité d'une esthétique dont le point d'honneur est de s'opposer à celle proposée en Occident (refus de l'art spirituel gratuit sans message politique, teneur des textes, manière de chanter, d'être sur scène...).

Les anciens militants ré-islamisés ont vieilli, les modes de prédication pour adolescents basés sur l'opposition (aux parents, à la société majoritaire) ne sont plus aussi efficaces. La SSI va donc apparaître comme un moyen de remobiliser sur de nouvelles valeurs (le plaisir personnel, le loisir, le professionnalisme...), les populations déçues par des acteurs islamistes ayant échoué dans les objectifs qu'ils s'étaient fixés (islamisation de la société, poids politique sur l'État français, revendications sur le respect des droits sociaux et culturels des musulmans tels que le port du voile, la lutte contre l'islamophobie...).

Les conférences de Tariq Ramadan (intellectuel suisse, à l'origine d'une grande part du processus de ré-islamisation de l'Europe francophone) commencent à changer de public au début des années 2000. Le profil du jeune musulman militant « recherchant la science » bloc-notes à la main, en vogue dans les années 1990, laisse place à celui de "fans" venus participer à un évènement hautement émotionnel, qui permet « d'augmenter sa foi » selon la formule consacrée. "Pour les conférences tout le monde est là, est bien habillé, se fait voir, mais en réalité plus personne n'a envie d'être sur le terrain... ", confie une ancienne militante ${ }^{13}$. Devant la déception suscitée par le militantisme politique traditionnel, émerge l'importance de nouveaux modes possibles de mobilisation non idéologisées selon les schèmes classiques des islamistes. Ainsi, Tariq Ramadan lance, en 2000, un grand concours sur l'art islamique, l'association de l'APAM «Promotion des Auteurs Musulmans ", appelant les jeunes musulmans d'Europe à soumettre leurs créations inspirées par l'islam, pièces de théâtre, poésies, dessins... Le succès mitigé rencontré par ce projet peut s'expliquer par son aspect alternatif, un contre-espace islamique qui maintient les musulmans hors monde. Les ré-islamisés vont percevoir la nécessité de répondre différemment aux attentes du public musulman engagé en inventant et proposant de nouveaux supports d'expression publique de l'identité islamique. La mobilisation ne va plus de soi. Les musulmans engagés ne répondront plus présents uniquement parce qu'ils sont musulmans. Il faut désormais séduire son public.

\section{Mobiliser après le 11 septembre : Rire, chanter, séduire}

L'incarnation de l'islam en diaspora, l'autorité de dire ce que doit être l'islam en Occident et dans le monde moderne, ne s'exprime plus seulement à travers les figures traditionnelles de l'imam ou du responsable associatif : un phénomène

13. Entretien avec O., 29 ans, ancienne militante, dentiste. 
de «staricisation » des conférenciers, rappeurs, chanteurs, comédiens ou encore acteurs et présentateurs télé du paysage islamique occidental, est à l'œuvre. Leur trait commun est d'être tous issus du monde islamique engagé et d'y avoir exercé des activités hautement légitimantes. Militants actifs et connus, responsables d'association, ils connaissent parfaitement la communauté islamique, sont connus d'elle, et cette expérience va servir de base à leur carrière dans la SSI. En son sein, ils se voient conférer une forme originale et inédite d'autorité religieuse, de mobilisation, d'organisation et de diffusion de l'identité islamique, non plus fondée sur un savoir purement religieux et intellectualisé, mais sur la notoriété conférée par leurs activités de représentation de l'islam public. Si la notoriété remplace aujourd'hui de plus en plus l'autorité religieuse, c'est que la staricisation de ces figures publiques est en grande partie due à cette nouvelle forme d'organisation et de mobilisation de la communauté musulmane après l'islamisme des années 1980 et 1990, qu'est la SSI.

Ces nouveaux modes d'expression mobilisent un public de consommateurs grâce au plaisir personnel qu'ils procurent, et au renouvellement de l'identité islamique qu'ils induisent. Un de leurs traits majeurs se retrouve dans l'humour et la dérision de l'histoire de l'islamisme et de ses codes. Des symboles normatifs puissants, jusqu'ici intouchables, sont mis en scène et permettent aux héritiers de l'islamisme de se repositionner en tant que musulmans engagés utilisant d'autres supports. Ainsi le label français de production "Connexion ", sponsorisé par la boisson soda "Muslim Up » et créé par les enfants d'un ancien responsable du mouvement islamiste tunisien (MTI), propose, sur son site Internet, ses services professionnels de graphisme et d'animation. Il y commercialise également, entre autres choses, des chansons pastiches.

"Moi Zoulikha » par exemple, chanté par un homme avec une voix stridente d'adolescente hystérique sur l'air du tube pop de la chanteuse française Alizée "Moi Lolita », se moque du profil « jeunes filles pieuses » se rendant en groupe à des conférences religieuses :

«Moi je m'appelle Zoulikha, avec mes copines tous les samedis on va/ écouter les conférences/ c'est super c'est vrai on arrive en avance/ pour mieux augmenter sa foi/ être au premier rang pour pouvoir écouter/ les conférenciers parler/ c'est super c'est vrai on sent plus de piété/ c'est pas de ma faute/ moi Zoulikha quand je vais làbas/ ma foi augmente/ les conférences sont à mon sens/ bonnes pour ma foi/ à moi/ moi Zoulikha...»

Le stéréotype "frère en qamis (habit reproduisant le style vestimentaire du prophète) et en Nike ", obsédé par le mariage, n'est pas en reste. Avec un accent banlieusard marqué, le groupe chante :

"C'est les reufrés (frères) 2003/ c'est ça/ en beubar (barbe) et en sunna (manière de s'habiller du Prophète)/ pour toi/ en Nike air et en Puma/ les reufrés du neuf trois/ vite Kader va chercher le cabriolet/ tous ensemble ce soir c'est le salon du Bourget/ une fois dans l'année on peut se laisser aller/ il faut aller trouver chaussure à nos 
pieds/ (...) bien la repérer tiens mets-toi sur le côté/ le hidjab est exigé/ je lui glisse un bout de papier...»

Loin d'être d'anecdotiques blagues de potaches, ce label a également produit un single "Femmes et croyantes" chanté dans le style R\&B (pop rhythm and blues) par une responsable de la Ligue française de la femme musulmane, affiliée à l'UOIF, afin de s'opposer à la loi d'interdiction du port du voile.

Aux États-Unis, les Muslim Students Associations s'en donnent également à cœur joie sur les campus en tournant des sketches vidéo diffusés sur Internet. Ces petites histoires se moquent, par exemple, de la difficulté de « la jeunesse musulmane » à se réveiller pour la prière (très) matinale du fajr en mettant en scène un super héros, faisant le tour des chambres avec sa cape afin de réveiller ses amis sur l'air du tube «Wake me up, before you go go ». Ils y critiquent le cliché islamiste de la non mixité à travers les impressionnantes acrobaties de la figure féminine de "ninja sister » en hijab de combat, chargée de veiller sur les bonnes mœurs du campus. L'obsession du pouvoir des leaders d'associations ne leur échappe pas. Ceux-ci sont présentés comme prêts à tout pour se faire élire à la tête de l'association, y compris à revêtir un niqâb (voile couvrant le visage) pour truquer les votes.

Pour ceux qui se rendent aux rencontres organisées, le one man show et les pièces de théâtre semblent également supplanter les prêches traditionnels des halaqât (Sunna [tradition] du Prophète, mais surtout cercles de discussion très utilisée par les Frères Musulmans dans le monde arabe), tajlîs (assemblées en petit comité, très pratiquées par le mouvement piétiste du Tabligh) et autres ijtimâ' (réunions), qui échouent à transmettre une identité islamique utile, correspondant de façon pragmatique aux défis posés par la justification de l'identité islamique en Occident face aux stigmates du terrorisme, de l'obscurantisme, ou face à sa célébration. Le succès rencontré par ces festivals d'art et de culture islamiques qui se multiplient aux États-Unis, en Angleterre, en Allemagne, à Lausanne, à Bruxelles ou en banlieue parisienne, est aussi le signe du renouvellement des modes de mobilisation islamiques, pour des populations qui se détournent de l'action accomplie grâce à la maîtrise de l'outil théologique ou du discours religieux savant, proposée par les islamistes. Plus encore, cette identité "savante» s'est entre temps retournée contre ses détenteurs au travers des discours post-11 septembre sur le nouveau danger terroriste émanant non plus des exclus mais d'universitaires issus des classes moyennes et "parfaitement intégrés ", ou de l'effroi provoqué par "l'intelligence fine et diabolique » de tel militant intervenant sur les plateaux de télévision lors des débats sur le port du voile. Les autorités islamiques traditionnelles elles-mêmes commencent à investir la SSI pour certains talk-show sur des chaînes locales (appartenant ici à la communauté noire africaine et antillaise). On peut y voir des imams débattrent entre deux clips de jeunes filles très légèrement vêtues. 
Si la SSI marche c'est qu'elle n'est ni coûteuse, ni lourde à assumer dans la vie quotidienne du musulman engagé. Sans les tensions des discours politiques, elle procure du loisir moral et familial et réconcilie les répertoires turc, ourdou, anglais, arabe, français, là où la réislamisation islamiste avait amené un éclatement du lien parents-enfants, ainsi qu'une supériorité arrogantes des repères arabophones. Elle procure également un cadre d'appartenance plus large et moins contraignant que les associations islamiques des premières heures, puisque ses supports sont partagés indépendamment de l'appartenance idéologique de chacun. On voit ainsi comment la matrice islamique de cette consommation culturelle, spécifiquement pour la musique, peut regrouper des acteurs aux adhésions très différentes tels que les rappeurs français Kerry James, membre du très controversé mouvement des Ahbach, et Abd al Malik, ancien tablighi tourné vers le soufisme, ou encore le chanteur anglais Cat Stevens, alias Youcef Islam, plus proche de la tendance Frères Musulmans. Elle permet même à certains groupes minorisés par la préséance de l'action politique pour obtenir la reconnaissance l'identité islamique, de ré-émerger. C'est le cas de ces « Bourda Nights » organisées aux États-Unis par des étudiants soufis (mais auxquelles participent toutes les tendances) pour chanter en groupe à la gloire du Prophète. La reconstruction du groupe des musulmans, engagés après le 11-septembre par l'intermédiaire de la SSI, permet de comprendre dans quel cadre se font les ruptures et les continuités avec l'héritage commun des codes construits par l'islamisme.

\section{Post-islamisme et désengagement}

Depuis le début des années 2000, l'entrée de l'islam dans l'espace public comme objet fréquent de débat court-circuite les réseaux traditionnels d'information des structures associatives militantes, jusque là principales actrices de la ré-islamisation. Certains nachîd sont désormais vendus au rayon spirituel de la FNAC, Tariq Ramadan se lit dans Le Monde, et c'est par la télévision que les musulmans apprennent l'initiative de l'UOIF de promulguer une fatwa (avis juridique) pour mettre fin aux "violences urbaines " de novembre 2005... Cette mise à disposition des musulmans engagés de la ressource islam dans l'espace public global, s'accompagne également d'un désengagement de leur part des structures islamiques en circuit fermé, c'est-à-dire prioritairement accessibles aux militants réguliers. En effet, un certain embourgeoisement par le travail de ces populations autrefois adolescentes ou étudiantes, donc hors de la vie active, fait qu'elles n'ont plus ni le temps ni l'envie de créer ou de consommer de la culture islamique très disciplinée avec des thèmes liés exclusivement à l'agenda idéologique du groupe concerné, au sein de structures associatives très hiérarchisées. Plus largement, il y a, passée la période de réislamisation, comme une réappropriation de l'islam militant jusqu'ici confisqué par les islamistes. Dieu n'appartenait qu'au groupe, il est désormais accessible à la consommation ou à la création individuelle d'une pièce de 
théâtre ou d'une cassette de nachîd hors du champ militant. Que devient alors l'identification aux expériences communes vécues tout au long des années 1980 et 1990, jusqu'alors constituantes du capital islamique, et en ayant instauré des codes, des valeurs et des répertoires d'action spécifiques?

Pour comprendre ces changements que nous appellerons post-islamistes et qui se déroulent aussi dans le monde arabe, certaines hypothèses sont avancées. Elles ne sont pas présentées ici de façon exhaustive et ne concernent pas le monde de la recherche anglophone (Eickelman, Piscatori, 1996 ; Beinin, Stork, 1997 ; Hafez, 2003 ; Wiktorowicz, 2004). Parmi elles, il y a celle qui, partant de l'échec de l'islam politique, de l'incapacité des islamistes à prendre le pouvoir politique, observe une dépolitisation relayée par une culture néofondamentaliste vide, se calquant sur la morale sans autre projet de société culturel ou politique (Roy, 2002). D'autres voient dans le déclin de cet islam politique, la promesse d'une ouverture démocratique et d'une réconciliation avec les répertoires politiques musulmans traditionnels (Kepel, 2002). Une deuxième explication opte pour un contournement de l'islamisme pour inventer de nouvelles formes d'islamisation apolitiques (Haënni, 2005). Une troisième explique ne pas vouloir surestimer le langage des acteurs en l'enfermant dans un cadre appelé islamisme qui primerait sur les autres cadres d'identification, identitaires et culturels (Burgat, 2005) et qu'il faudrait plutôt replacer dans son environnement historique (le nationalisme arabe par exemple) (Roussillon, 2005).

Partant de ces hypothèses, il est alors possible de proposer un changement de paradigme qui permette de comprendre le rôle de l'islamisme dans la militance engagée aujourd'hui. En effet, ces interprétations ont en commun de positionner l'islamisme dans une situation de rupture avec la tradition, culturelle ou politique. Il faut cependant prendre en compte la façon dont l'islamisme forge lui-même désormais une tradition à part entière. L'islamisme, nous l'avons vu plus haut, fait partie du capital islamique des acteurs musulmans engagés en Occident. Depuis le début des années 2000, ces derniers ne se positionnent pas exclusivement vis-à-vis de l'islamisme par la rupture, la disqualification ou le contournement. Ils y voient également une tradition, un moment historique capable de fédérer une communauté, délivrant un sentiment d'appartenance grâce aux expériences passées communes (qu'elles aient été vécues directement ou indirectement). Ils se situent donc aussi en continuité avec cet héritage qu'ils considèrent à la fois positivement ou négativement.

\section{La contestation du « vrai islam » islamiste}

«Tu vis chez tes parents, à un moment tu reçois la révélation, tu te prends pour un prophète et tu vas dire à tes parents l'islam c'est ça. Moi mes parents m'ont transmis des choses qui n'étaient pas intellectuelles genre Tariq Ramadan, mais qui m'ont fondé. J'ai fait des grosses conneries, et je suis revenu sur le droit chemin. À l'époque je l'ai attribué au "vrai islam”, mais je me rends compte que c'est un tout. Nos parents, 
même la société, nous ont transmis des valeurs qui nous permettent d'être ce qu'on est. C'était absent dans nos analyses, on voyait que la forme, genre y'a tout dans le Coran, le reste c'est des sciences de koufars (mécréants) »(F., 37 ans, musicien et militant associatif).

Les discours sur la découverte du "vrai islam »par opposition à l'islam négatif des parents, importé des pays d'origine et dénaturé par les traditions qui y ont cours, ou encore l'opposition à certaines traditions islamiques folklorisées et euphémisées par l'espace public comme le soufisme, ont été un moment important de la ré-islamisation islamiste des jeunes musulmans. Désormais, ces acteurs ont aussi une réflexion sur les codes constitués, petit à petit, au sein de ce "vrai islam ", telle que la rupture avec les parents et leurs traditions religieuses. Le mécanisme de défense culturelle d'une identité militante pure, menacée par l'éloignement de la lecture islamiste des normes, laisse la place à un travail de liaison entre les expériences islamistes, les valeurs séculières de l'espace public et l'islam des parents. Les nachîd au sein de la SSI ne servent plus à glorifier le groupe d'élus et ses expériences spécifiques, mais à louer sur un son de guitare sèche la figure des mamans en accord avec les valeurs islamiques tempérées de respect des parents. Telle pièce de théâtre s'inspirant d'un récit coranique servira avant tout à dénoncer les injustices subies par les enfants dans le monde et non plus à plaider la cause exclusive des enfants de Palestine, de Tchétchénie ou de Bosnie.

"Ca sert à rien de saouler les gens avec "l'islam c'est la vérité", etc. Nous on sait ce qui nous inspire, c'est fort. Maintenant on va pas saouler les gens, on n'est pas des savants. Et puis on peut pas tout dire dans une pièce c'est le débat qu'on suscite après qu'est intéressant (...) Même avec la guerre des étoiles on peut trouver des trucs islamiques ! Dark Vador, le bien, le mal, l'apprentissage... » (A., comédien, 29 ans)

Il faut considérer l'importance du déroulement historique de l'islamisme. Le fait qu'il existe désormais une vieille génération d'islamistes comme il existait, au début des années 1980 et 1990, une génération de parents que l'on pouvait critiquer, permet des ruptures et des remodulations de l'identité et du militantisme islamiques publics. Depuis le 11-septembre, les jeunes musulmans veulent plus que jamais lutter contre cette assignation identitaire qui fait de chaque musulman engagé, possédant l'héritage de certains codes utilisés par l'islamisme, un islamiste lui-même. Ils semblent également victimes d'une certaine fatigue militante et idéologique et d'une nécessité de se réinventer en tant que musulmans engagés. La routinisation et la massification des codes islamistes (le port de la barbe ou le voile librement choisi en sont un exemple ${ }^{14}$ ) leur a fait perdre leur caractère

14. La réalité historique de l'islamisme reste (c'est une ressource créée à un moment, qui différencie de la masse des musulmans, qui doit être protégée, et qui permet une reconnaissance entre ses membres). Cependant son usage social est complètement réinventé. On peut voir ainsi de plus en plus de jeunes femmes porter un voile qui couvre leurs cheveux mais accompagné d'une jupe qui arrive aux genoux. Concernant ces nouveaux acteurs, l'explication d'une pensée islamiste qui perdure et dont les nouveaux habits seraient une ruse stratégique, n'est pas satisfaisante. Ils sont certes dans une continuité avec l'identification à un certain type d'islamité engagée, mais cet héritage s'exprime dans un cadre historique et traditionnel de l'islamisme, au regard duquel de nombreux codes sont réinventés. 
innovant. La forme est devenue plus importante que le fond, passant à la trappe la justification d'authenticité qui était à la base du mouvement islamiste.

Les musulmans engagés se repositionnent non plus vis-à-vis des parents comme le faisait le «vrai islam ", mais vis-à-vis de leur propre parcours militant. Il y a aujourd'hui une crise de la transmission des valeurs et des récits islamistes à de jeunes générations qui ont finalement un capital islamique aux sources diversifiées, dont la cohérence est réinventée et qui amène par la même à repenser l'obligation de passer par les biographies islamistes classiques pour pouvoir agir publiquement et de manière engagée avec la référence islam.

\section{La contestation de la biographie islamiste}

"C'était genre le mec arrivait : "non c'est pas comme ça vous connaissez rien au dine (la religion), c'est du n'importe quoi ce que vous faites!”, il avait une barbe, il te plaçait deux hadiths (dires du Prophète) et trois aya (versets) en arabe et toi ça y est t'étais subjugué. En fait c'était beaucoup de bruit pour rien... » (A., comédien, 29 ans)

La réconciliation des jeunes musulmans avec l'espace public global et l'atténuement des tensions vis-à-vis de la culture des parents ou de la culture de préconversion à l'islam militant (la culture beur), donnent à voir un travail de mise en récit de la cohérence biographique de ces jeunes pour lesquels l'islamisme ne représente qu'une étape et non plus l'aboutissement de l'expérience islamique. Dans ces récits, les codes de l'islamisme qui voulait faire tabula rasa des traditions religieuses et idéologiques "non conformes ", sont désormais inclus dans un universel où toutes les traditions peuvent servir de base à l'expérience islamique. Le fait qu'une mémoire, un passé historique de l'islamisme existe désormais, permet d'en faire une tradition et, par là même, de s'inventer un avenir différent qui ne passe plus par une rupture violente.

L' "Islamic way of life " promu par les islamistes jusqu'au milieu des années 1990 ne disparaît pas totalement, mais il n'est plus le seul cadre de référence. D'autres configurations permettent un accès à l'expérience islamique engagée. C'est le cas de l'éthique notamment, à travers la question du professionnalisme, érigé en véritable norme religieuse, car le musulman doit avoir une production de haute qualité. Le caractère paisible des productions culturelles sans agressivité ni références au sexe, les valeurs du travail et de l'honnêteté font partie de cette éthique qui permet de réinventer les codes de respect de la norme islamique. Bien sûr, l'appartenance à un moment donné au mouvement ou à la pensée islamiste reste un élément de légitimité fort, mais c'est une légitimité historique et non plus idéologique et effective, l'islamisme devenant une tradition. Une nouvelle source de légitimité est désormais activée : l'authenticité. Se réinventer une lignée croyante (Hervieu-Léger, 1997), permet de choisir son avenir et de le fabriquer rationnellement. Aujourd'hui cet avenir est très marqué par le souhait d'une réussite sociale dont l'éthique est la base. Devant cette dilution des codes islamistes dans un cadre éthique plus large, les acteurs de la SSI créent une sorte 
de discours conversionniste. Pour eux, ils redeviennent ce qu'ils n'ont jamais cessé d'être, des musulmans. Ils font de l'islamisme une étape, et relient l'adoption de l'éthique à des valeurs qu'ils auraient toujours possédées. L'intérêt pour l'art est alors réactivé dans les récits biographiques comme un intérêt pour l'universel dont l'islam n'a été qu'un support. La majorité des acteurs de la SSI ont, en effet, joué dans des groupes de rap ou de blues, ils ont fait du théâtre ou d'autres activités artistiques, avant, voire pendant leur réislamisation. Cependant, ils n'assumaient pas ces activités comme faisant partie de leur capital islamique, ce qui a permis à la SSI de devenir une contribution à la culture globale. Sa dimension apparemment non militante et non revendicative lui permet d'attirer un public ou des partenaires professionnels non musulmans. L'islam devient un atout supplémentaire, une plus-value, dans les interconnexions avec l'espace public et non plus un processus contraignant de mise à l'écart. C'est ainsi que s'explique le refus des organisateurs de Rythm'n Nacheed d'accepter d'être sponsorisés par une association caritative en faveur de la Palestine. Les artistes restent toujours sensibles à la question palestinienne mais les codes de l'islamisme sur cette question restreignent les modes d'action possibles. Aujourd'hui l'art devient pour eux un métier, avec une vraie force de mobilisation et une dimension engagée, intrinsèque à l'éthique de paix et de justice de l'islam.

\section{D'autres modalités d'appartenance et de partenariat}

«Le fi sabil lillah (faire les choses dans la voie de Dieu, gratuitement) c'est ce qui tue la communauté, le bénévolat ne dure pas, il faut qu'on se réveille et qu'on fasse les choses proprement ! Il ne faut pas que les gens soient des victimes de la consommation de masse, et qu'après avec les musulmans ils comptent au sou près. Il faut qu'ils nous prennent au sérieux comme n'importe quel artiste, c'est un problème de mentalité ! » (N., comédienne, animatrice radio et manager, 22 ans).

Une des caractéristiques de la SSI est d'inventer d'autres modalités d'appartenance et de participation à l'institution islamique que celles proposées dans les années 1990 (i.e. les mosquées, les associations, les évènements tels que les conférences ou les rencontres...). Cette participation promettait un salut lointain et impalpable là où désormais la SSI propose du beau, du loisir et un bien-être immédiatement consommables. On passe ainsi d'une population de militants à une population d'usagers, de clients. Il faut désormais séduire son public, proposer ses services à travers le loisir, car le lien d'interdépendance, de solidarité et de responsabilité des musulmans engagés ne fait plus recette.

Les acteurs de la société islamique continuent donc de maintenir des relations avec certaines institutions islamiques, mais elles ne sont plus la base exclusive à partir de laquelle se créent leurs actions, fussent-elles des actions islamiques. Le modèle d'action pour la sensibilisation et la mobilisation des usagers autour d'un art engagé que proposent les acteurs de la SSI, se retrouve dans des activités moins contraignantes et plus ludiques souvent gérées en leur nom propre, sans intervention d'une institution (ce qui n'empêche pas pour autant l'institution de gagner 
en légitimité par son partenariat avec l'acteur de la SSI). Fondant leur maison de production, donnant des cours de théâtre ou animant des ateliers d'écriture, les acteurs de la SSI visent à promouvoir l'émergence de générations nouvelles grâce à l'éthique d'un "islam conscient », faisant de la prévention antidrogue dans les écoles ou s'associant à la lutte contre la violence ou le racisme religieux...

Le lien élites/masse de la tradition islamiste au sein des institutions, se déplace vers une forme originale de ré-institutionnalisation de l'autorité et de soutien à la cause islamique. Les usagers participant aux actions proposées par la SSI le font souvent sous la forme du fan club (notamment à travers les sites internet des artistes concernés), où la notoriété de l'acteur de la SSI engendre chez eux l'adhésion et le soutien à la cause qu'il défend. Cette notoriété fondatrice d'un nouveau type d'autorité éthique concurrence les mécanismes d'allégeance au savoir cognitif et normatif des élites islamistes. Prendre des photos avec son cheikh préféré, son chanteur préféré voire son comique islamique préféré, est désormais une attitude bien plus commune pour matérialiser son islamité que de compiler les manifestes de Sayyid Qutb (penseur égyptien des Frères Musulmans). De la même façon, la notoriété de tel acteur incitera plus à faire un don à une œuvre de charité ou à faire partie d'un réseau d'associations, que la simple injonction normative de solidarité basée sur le corpus religieux. Le bien-être personnel qu'y trouvent les usagers leur est en effet préférable à l'utopique efficacité politique d'un bien-être collectif décidé d'en haut par des élites islamistes sévères passant leur temps à leur reprocher leur manque d'engagement.

Ces nouveaux modes d'action ont pour conséquences de nouveaux modes de partenariat pour les acteurs de la SSI. Ils préfèrent désormais l'action sous forme de réseaux, à la collaboration verticale et très hiérarchisée des institutions islamiques traditionnelles. Le nombre d'acteurs non musulmans impliqués dans ces partenariats est par ailleurs de plus en plus important, le primat étant donné à l'efficacité. Vont-ils finir par avoir leur agent, leur book, leurs tournées, là où les autres élites plus traditionnelles auront leur organisation et leur conseil d'administration?

Cette remise en question des frontières du groupe engagé et de ses partenariats le pousse à remettre en cause ses cadres d'action exclusivement intra-islamique, ce qui pose alors la question du partage des valeurs majoritaires. Si une certaine islamophobie en Europe, et particulièrement en France, limite ces ambitions de participation à l'espace public, aux États-Unis le sentiment d'appartenance nationale des musulmans engagés leur permet de dépasser le cadre communautaire d'action sans en abandonner la référence religieuse. On peut ainsi citer l'exemple d'un showman américain et ré-islamisé, dont le spectacle portant sur les réactions de peur post-11 septembre ${ }^{15}$, a été joué après le passage dévastateur de l'ouragan Katerina aux États-Unis, et dont les recettes ont été reversées aux populations

15. L'acteur porte une longue barbe qui charrie à elle seule le stéréotype islamiste et parle de la surveillance du FBI, des agressions sur les musulmans aux États-Unis... 
touchées, sans qu'il soit fait mention de l'appartenance religieuse des bénéficiaires. Dans la même veine, en Angleterre, Youcef Islam a composé une chanson au profit des victimes du tsunami qui n'était pas intitulée "venez en aide à vos frères musulmans " mais sobrement "Indian Ocean ». Il la présentera comme sa contribution en tant qu'artiste. Encore une fois, l'élément religieux n'est pas omniprésent.

La SSI cherche ainsi à imposer en douceur la plus-value de ce nouvel islam éthique et sa participation à l'espace public global. Elle veut aussi imposer de nouveaux codes compétitifs et majoritaires à la communauté musulmane elle-même, afin de la sortir du bricolage et de l'instabilité des différentes références non assumées. Le fi sabil lilah, par exemple, est réinterprété comme une action vers Dieu qui doit être de qualité. Son sens traditionnel de gratuité est fustigé par les acteurs de la SSI qui y voient une menace pour la qualité de leur production. Ils souhaitent exacerber des liens professionnels fondés sur une exigence de professionnalisme pour mieux servir l'islam, que la seule concordance idéologique ne saurait garantir. On passe ainsi d'une communauté de frères à une communauté de partenaires. Le caractère transitoire et intenable par définition en Occident, de la situation de défense d'une identité musulmane menacée par les valeurs occidentales a amené à repenser la pérennité des formes de mobilisations islamistes. Les musulmans engagés qui participent à la SSI ne veulent pas l'accomplissement d'un projet holiste d'une société islamique où les musulmans dominent. Ils souhaitent le règlement, ou l'allègement, de problèmes personnels correspondants à leur situation particulière, celle de classes moyennes occidentales.

Malgré une certaine diffusion transnationale de ses modes d'action (ÉtatsUnis et Europe bien sûr, mais aussi monde arabe et sud asiatique...), la SSI n'est pas homogène dans ses thèmes et ses visées. Ils dépendent beaucoup des cadres nationaux de la visibilité accordée à l'action islamique. Dans le monde arabe, la SSI euphémise ses revendications politiques "pures" afin de se fondre dans l'héritage islamique de la nation, tout en changeant les habitudes de mobilisation des musulmans qui consomment ce type de production sous la forme du mouvement social et hors des institutions étatiques. Ainsi, lors de l'épisode des caricatures du prophète Mohammad, la chanson Ana Assif ya Rassoul Allah (je suis désolée oh prophète de Dieu), pouvait passer en boucle sur les chaînes nationales du monde arabe tout en condamnant de façon non agressive le "laisser-faire " des musulmans du monde entier. La chaîne de télé Alafassiya créée et principalement animée par un prédicateur koweïtien, Al-Affassi, rappelle également en chanson les bienfaits de la prière et d'une épouse pieuse, mais se garde de critiquer les politiques nationales menées par les pays du Golfe lors de la guerre au Liban. On retrouve chez tous les acteurs qui recourent à la SSI, où qu'ils soient, une même volonté de renouveau du système de références qui maintient certaines des bases posées par l'islamisme. Le réseau devient donc la forme d'organisation privilégiée pour ces phénomènes de pluri-appartenances souples, ou l'identité islamique est célébrée selon le degré d'accès au politique, produisant une relation 
structurelle entre ce que l'on veut défendre et la forme spécifique de mobilisation que l'on emploie pour cela.

La SSI permet à la fois d' " être un musulman normal, qui met du gel » ${ }^{16}$, et d'avoir une position de production de sens privilégiée au sein de la communauté engagée. Position consolidée par la disparition de la volonté de convaincre et de démontrer selon la façon agressive du militant traditionnel. La section appelée daawa (prédication) sur le website de Youssef Islam se contente ainsi de renvoyer à des organisations pour des réponses sur l'islam et une meilleure connaissance de ce qu'est l'islam. On n'y trouve pas d'injonction normative, juste une invitation à la découverte. Il semblerait que l'islam ait pris suffisamment de place dans la sphère publique (y compris à cause de sa mauvaise réputation), pour faire disparaître chez le musulman engagé l'envie d'en faire trop.

Cependant les témoignages de volonté de mise à niveau et d'égalité de traitement des acteurs publics islamiques avec les autres acteurs de l'espace public global et séculier, ne doivent pas occulter la visée alternative de ces nouveaux modes de mobilisation. La SSI est là où on ne l'attend pas, elle s'aligne sur les valeurs et les cadres dominants mais son but est de les concurrencer de l'intérieur. Ainsi, lors du réveillon du 31 décembre 2004, 2005 et 2006, des conférenciers de la région parisienne ont décidé de proposer des sketches et conférences ludiques dans certaines mosquées européennes afin de dissuader les jeunes musulmans de réveillonner de façon peu licite. On note le même type d'initiative au sein de la Muslim Students Association de New York University où le jour de la St Valentin 2007 fut prétexte à une veillée spirituelle sur la place de l'amour en islam. Aujourd'hui, la culture islamique n'est pas encore prise au sérieux par les médias et par la majorité des leaders musulmans des anciennes générations. Son combat pour devenir une référence à grande échelle n'est donc pas encore gagné. On l'interprète souvent superficiellement comme la main rampante d'un islamisme politique ${ }^{17}$ pour qui elle est un prétexte à l'endoctrinement, ou bien encore on se moque de son aspect folklorique n'en retenant que le cliché misogyne d'adolescentes voilées se "déhanchant » ${ }^{18}$ lors des concerts. Cependant, la façon dont elle « habilite» ses consommateurs en leur démontrant qu'ils sont capables d'être des musulmans engagés, sans intermédiaires religieux explicatifs, qu'ils soient normatifs, textuels ou politiques, pourrait annoncer son succès grandissant et sa pérennité.

\section{Amel BOUBEKEUR EHESS, Paris amel.boubekeur@wanadoo.fr}

16. Entretien avec J., chanteur au sein d'un groupe de nachîd modernes, 24 ans. Avoir une apparence physique trop à la mode était en effet contraire aux codes esthétiques initiés par l'islamisme.

17. Voir, par exemple, la teneur de l'article "L’islam radical investit dans la mode ado », Le Figaro, 08/09/2005.

18. "La musique “islamiquement” correcte en concert à Marseille », Libération, 20/12/2004. 
Abd al Malik, 2004, Qu'Allab bénisse la France! Paris, Albin Michel.

AMghar Samir, 2003, « Rap et Islam : quand le rapeur devient imam », Hommes et migrations, 1243.

BAYAT Asaf, 2005, "What is post-islamism ? ", ISIMNewsletter, 16.

Beinin Joel and Stork Joe (eds.), 1997, Political Islam, Berkeley, University of California Press.

Bennani-Chraï̈i Mounia, Fillieule Olivier, 2003, Résistance et protestations dans les sociétés musulmanes, Paris, Presses de Science Po.

Boubekeur Amel, 2005a, "Cool and Competitive. New islamic culture in the West ", ISIMNewsletter, 16.

-, 2005b, "L'islam est-il soluble dans le Mecca Cola ? Marché de la culture islamique et nouveaux supports de religiosité en Occident ", Maghreb-Machrek, 183.

-, 2006, "L'islamisme comme tradition : fatigue militante et désengagement des élites islamistes ", in Amghar Samir (ed.), Islamismes d'Occident. État des lieux et perspectives, Paris, Lignes de repères.

Burgat François, 2005, L'islamisme à l'heure d'al-Qaida, Paris, Éditions La Découverte. Debord Guy, 1967, La société du spectacle, Paris, Gallimard.

Eickelman Dale, Piscatori James, 1996, Muslim Politics, Princeton, Princeton University Press.

Göle Nilüfer, 2002, "Islam in Public: New Visibilities and New Imaginaries ", Public Culture, 14-1.

HaËNNI Patrick, 2005, L'islam de marché, Paris, Seuil.

HafEz Mohammed, 2003, Why Muslims Rebel ? Boulder, Lynne Rienner.

Hervieu-LÉGer Danièle, 1997, "La transmission religieuse en modernité : éléments pour la construction d'un objet de recherche ", Social Compass, 44-1.

KePel Gilles, 2000, Jihad, expansion et déclin de l'islamisme, Paris, Gallimard.

KePel Gilles, Yann Richard, 1990, Intellectuels et militants de l'islam contemporain, Paris, Seuil.

Khosrokhavar Farhad, 1997, L'islam des jeunes, Paris, Flammarion.

Laurens Henri, 2000, L'Orient arabe. Arabisme et islamisme de 1798 à 1945, Paris, Armand Colin.

Roussillon Alain, 2005, "Dans l'attente du post-islamisme", La vie des idées, novembre 2005.

RoY Olivier, HAËNNI Patrick, 1999, "Le post-islamisme », Revue des mondes musulmans et de la Méditerranée, 85-86.

Roy Olivier, 1992, L'échec de l'islam politique, Paris, Seuil.

-, 2002, L'islam mondialisé, Paris, Seuil.

Tilly Charles, 1978, From Mobilization to Revolution, Boston, Addison-Wesley.

WikTorowicz Quintan (ed.), 2004, Islamic activism. A Social Movement Theory. Bloomington, Indiana University Press. 


\section{Résumé}

L'analyse des mobilisations politiques s'inspirant de l'islam est souvent indissociée des questions de violence, de modernité et d'(in)compatibilité avec la culture occidentale. Depuis peu, on assiste néanmoins chez les musulmans engagés en Europe et aux USA à l'émergence d'un nouveau type d'islam militant, notamment à travers des mobilisations artistiques et culturelles, questionnant fortement ces perceptions.

Pendant longtemps, le militantisme inspiré de l'islamisme a représenté la principale ressource politique pour mobiliser autour de la question Islam. Aujourd'hui de nombreux militants européens abandonnent les meetings et conférences inspirées de la matrice de l'islamisme traditionnel et choisissent de prêcher et de diffuser la norme islamique grâce à de la pop music, des sketchs et pièces de théâtre, des bandes dessinées ou des émissions de télévision. Cet article tente de décrire et d'analyser les liens et tensions existants entre le militantisme islamiste traditionnel et ces nouvelles formes de mobilisations artistiques et culturelles. On pourra ainsi comprendre le sens des récentes transformations du militantisme islamique en Europe et la manière dont elles reconfigurent l'islam politique en tant que tel.

Mots-clés : islamisme, islam de France, culture, militantisme, espace public.

\section{Abstract}

Political mobilisations inspired by Islam are often seen as linked to violence, lack of modernity and incompatibility with the western culture. However the recent emergence in Europe and the US of a new type of militant Islam, especially through arts and culture, is challenging these perceptions. For a long time, Islamist political militancy has been the principal means to mobilize politically engaged Muslims around Islam. Today, more and more of them abandon the meetings and political groupings of classical Islamism; and choose pop and rap music, sketches and theatre, television, comic strips, radios and television stations as new repertoire of action in order to preach, to affiliate, to diffuse norms and values or transmit Islamic identity.

This article tends to explain what does this tension between the traditional Islamist militancy and these new forms of cultural and artistic mobilization tell us about recent transformations of the politically engaged Muslims as well as about the reinvention of political Islam itself.

Key words: islamism, Islam in France, culture, militancy, public space.

\section{Resumen}

El análisis de las movilizaciones políticas que se inspiran en el Islam permanece a menudo estrechamente asociado de las cuestiones de violencia, de modernidad y de (in) compatibilidad con la cultura occidental. Desde hace un tiempo, asistimos sin embargo entre los musulmanes comprometidos en Europa y en los Estados Unidos a la emergencia de un nuevo tipo de Islam militante, que cuestiona fuertemente estas percepciones, especialmente a partir de movilizaciones artísticas y culturales.

Durante un largo periodo, la militancia inspirada en el islamismo ha representado el principal recurso político para movilizar alrededor de la cuestión del Islam. Hoy, numerosos militantes europeos abandonan las reuniones y las conferencias inspiradas en la matriz del islamismo tradicional y eligen rezar y difundir la norma islámica a 
I 38 - ARCHIVES DE SCIENCES SOCIALES DES RELIGIONS

partir de la música pop, de sketches y obras de teatro, de historietas o de emisiones televisivas. Este artículo intenta describir y analizar los lazos y las tensiones existentes entre la militancia islamista tradicional y estas nuevas formas de movilizaciones artísticas y culturales. Podremos asi comprender el sentido de las recientes transformaciones de la militancia islámica en Europa y la manera en que éstas reconfiguran al Islam político en tanto que tal. (Trad. de Véronica Béliveau-Giménez)

Palabras clave : islamismo, islam en Francia, cultura, militancia, espacio público. 